\title{
Unique Degradation Signatures of Organic Solar Cells with Non-Fullerene Electron Acceptors
}

\author{
Pranab Deb, Ryan T. Grimm, John K. Grey*
}

Department of Chemistry, University of New Mexico, Albuquerque, NM 87131

*jkgrey@unm.edu

SUPPORTING INFORMATION 


\section{Linearity check of IMPS response}

To verify the linearity in IMPS response, we performed IMPS measurements on devices exposed to different levels of aging where the average intensity was held constant, but the modulation depth varied between $10-100 \%$. We did this experiment at several intensities spanning the range reported in the original manuscript. The modulation depth dependent IMPS spectra (Nyquist plots) are shown in Figure S1-S3. Here we would like to mention that the modulation dependent studies were performed on a device which was different form the device mentioned in the main text. However, the overall experimental trend is similar in both cases. For pristine and lightly aged devices, the low frequency photocurrent $(<50 \mathrm{kHz})$ response maintains linearity at all modulation depths for the entire intensity range used in this study (Figure S4 \& S5). The DC photocurrent (frequency independent part of the photo current) disappears in case of heavily aged device due to the significant increase of the trap states and that is why we plotted the gain peak maxima (Figure 4a of the main manuscript) with intensity for heavily aged device. The variation of photocurrent gain peak maxima with respect to the excitation intensity is nonlinear (Figure S6). However, photocurrent dependency on the excitation intensity is similar at all modulation depths (Figures S4-S6). We also noticed that the photocurrent response is linear to the AC modulation depths for the entire intensity range at all device conditions (Figure S7). 

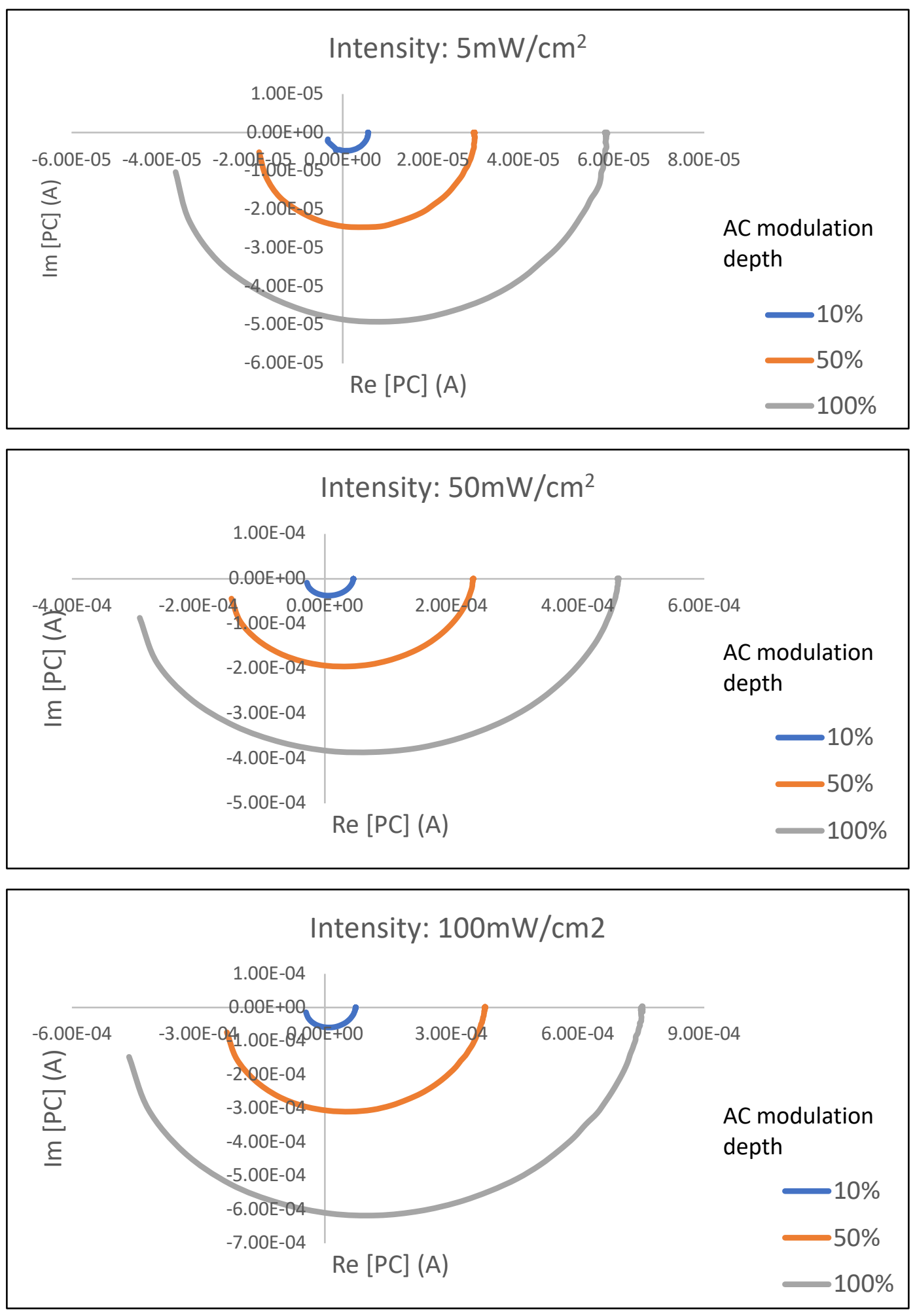

Figure S1: AC modulation depth dependent IMPS spectra (Nyquist) of a pristine PTB7:Coi8DFIC(1:1) device at 5, 50 and $100 \mathrm{~mW} / \mathrm{cm}^{2}$. 

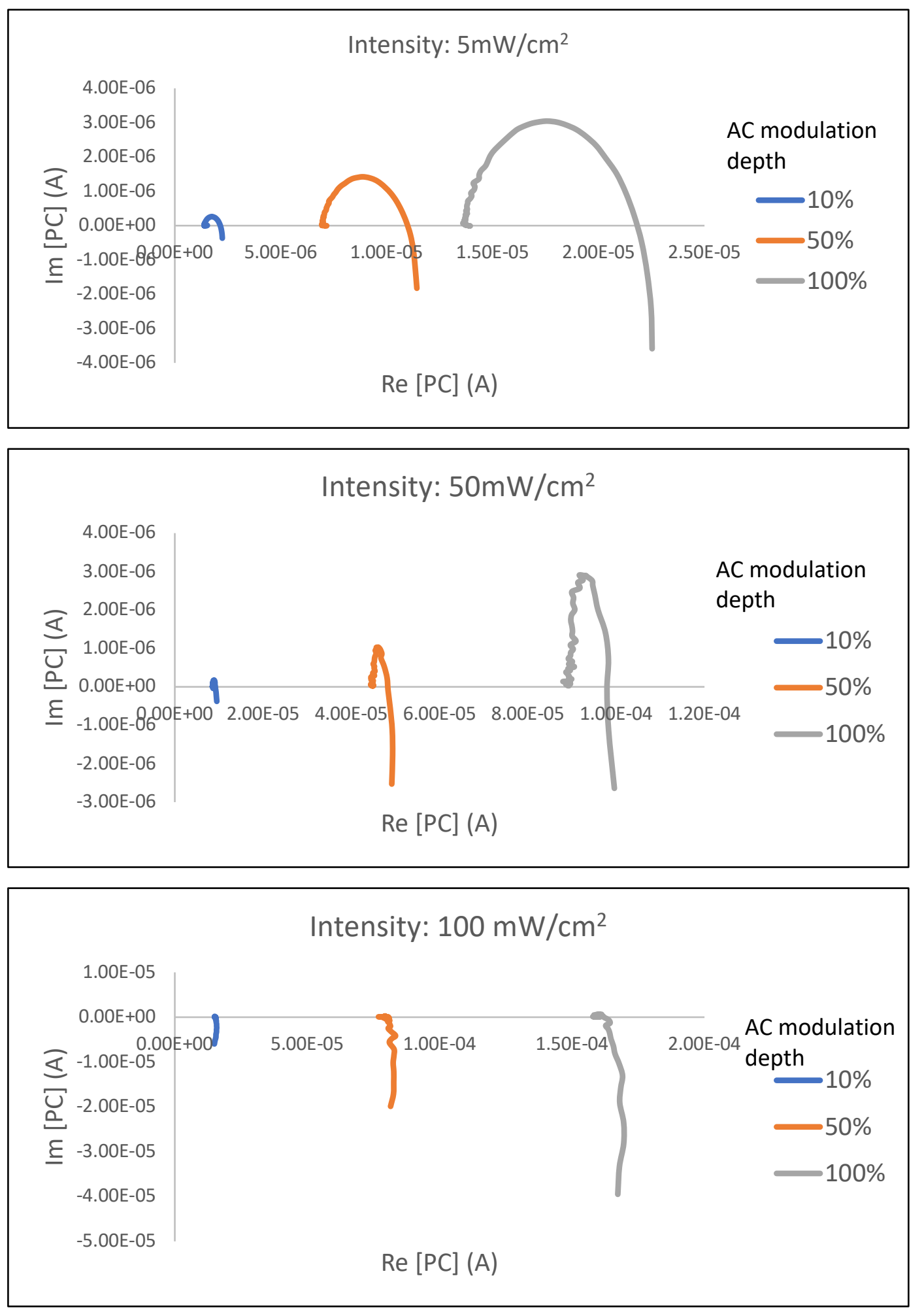

Figure S2: AC modulation depth dependent IMPS spectra (Nyquist) highlighting the $1^{\text {st }}$ quadrant of a lightly aged PTB7:Coi8DFIC(1:1) device at 5, 50 and $100 \mathrm{~mW} / \mathrm{cm}^{2}$. 

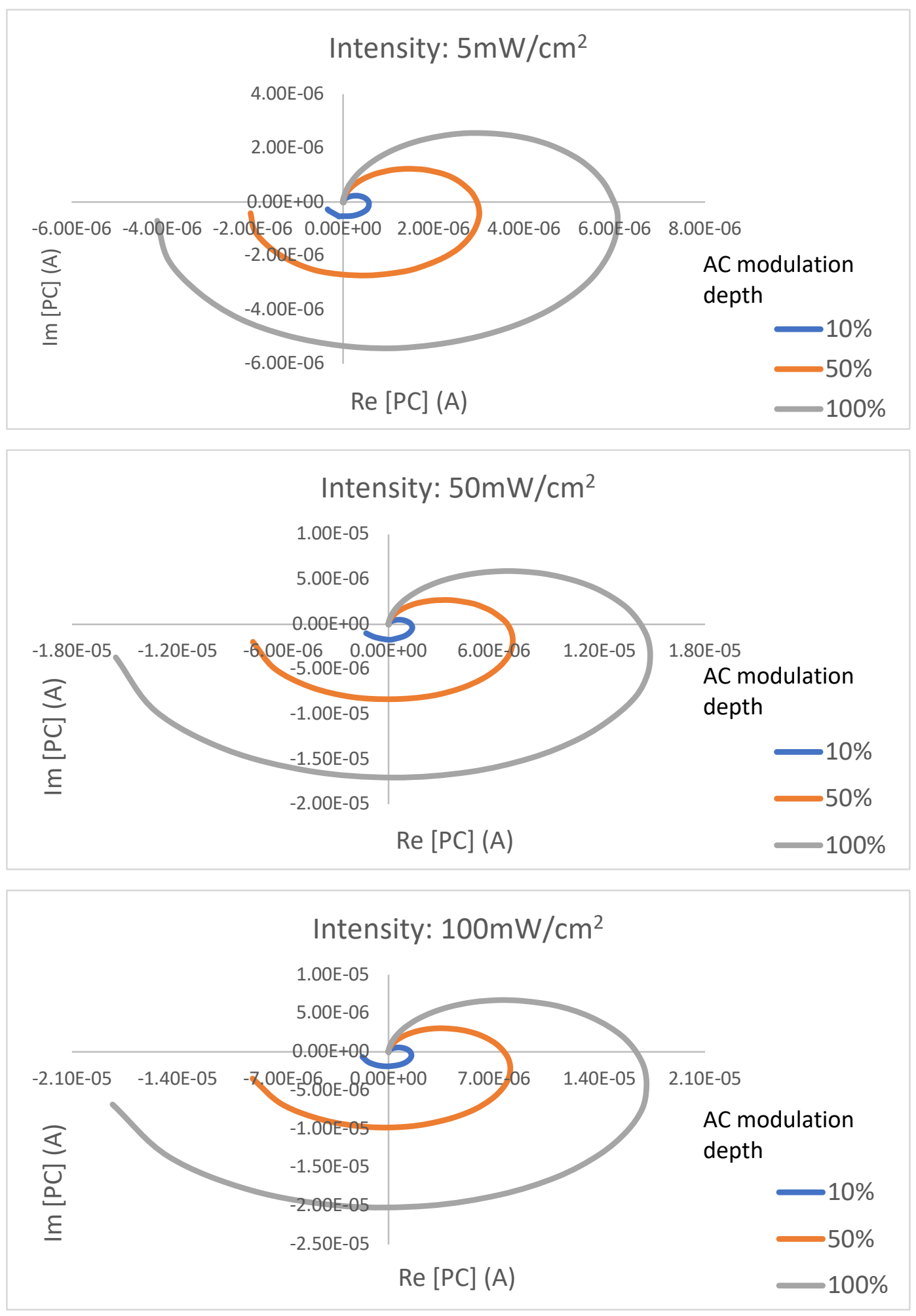

Figure S3: AC modulation depth dependent IMPS spectra (Nyquist) of a heavily aged PTB7:Coi8DFIC(1:1) device at 5, 50 and $100 \mathrm{~mW} / \mathrm{cm}^{2}$. 

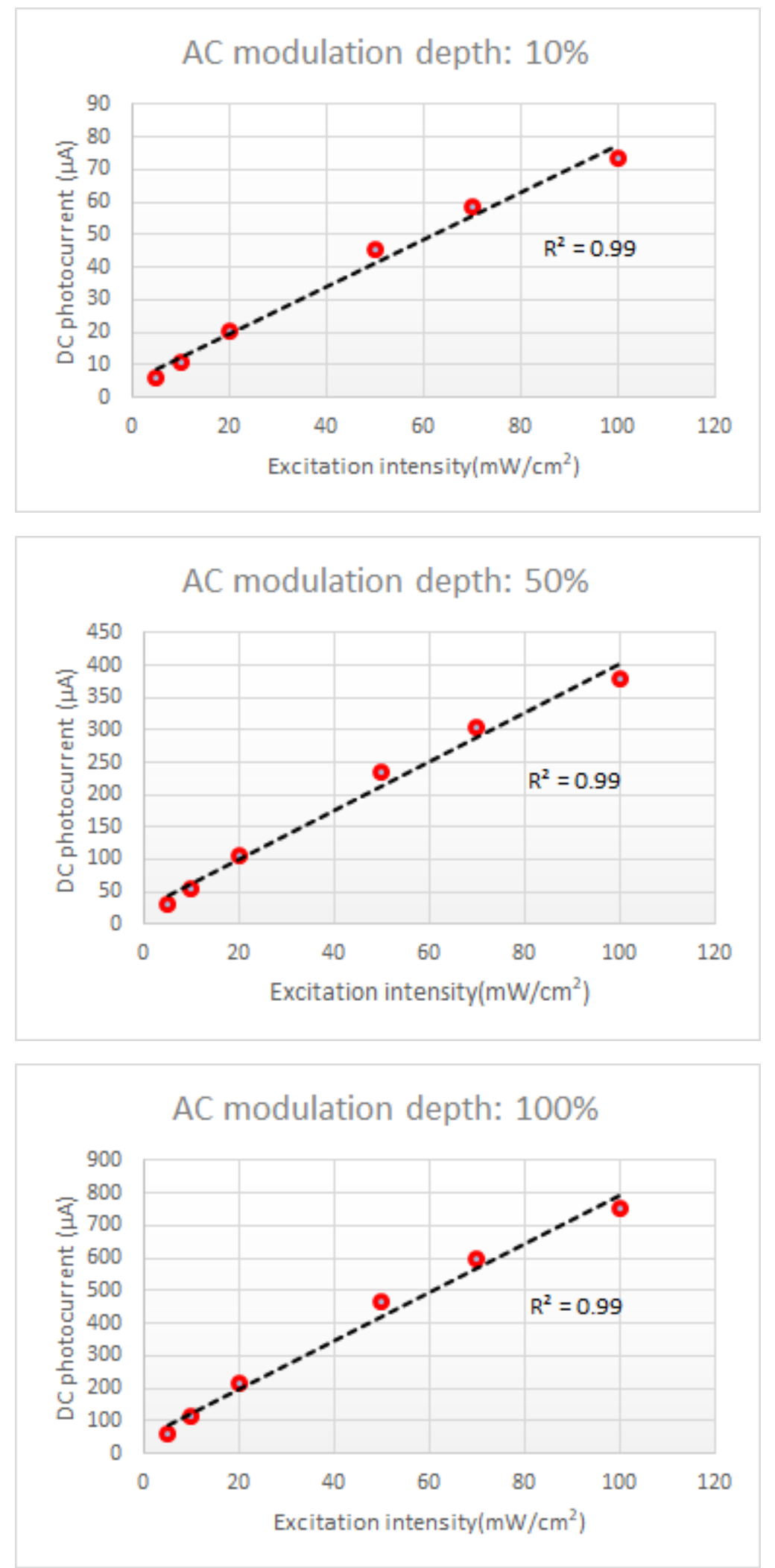

Figure S4: Intensity $\left(5,10,20,50,70\right.$ and $\left.100 \mathrm{mw} / \mathrm{cm}^{2}\right)$ dependent DC photocurrent of a pristine PTB7:Coi8DFIC(1:1) device at different depth of AC modulation. 

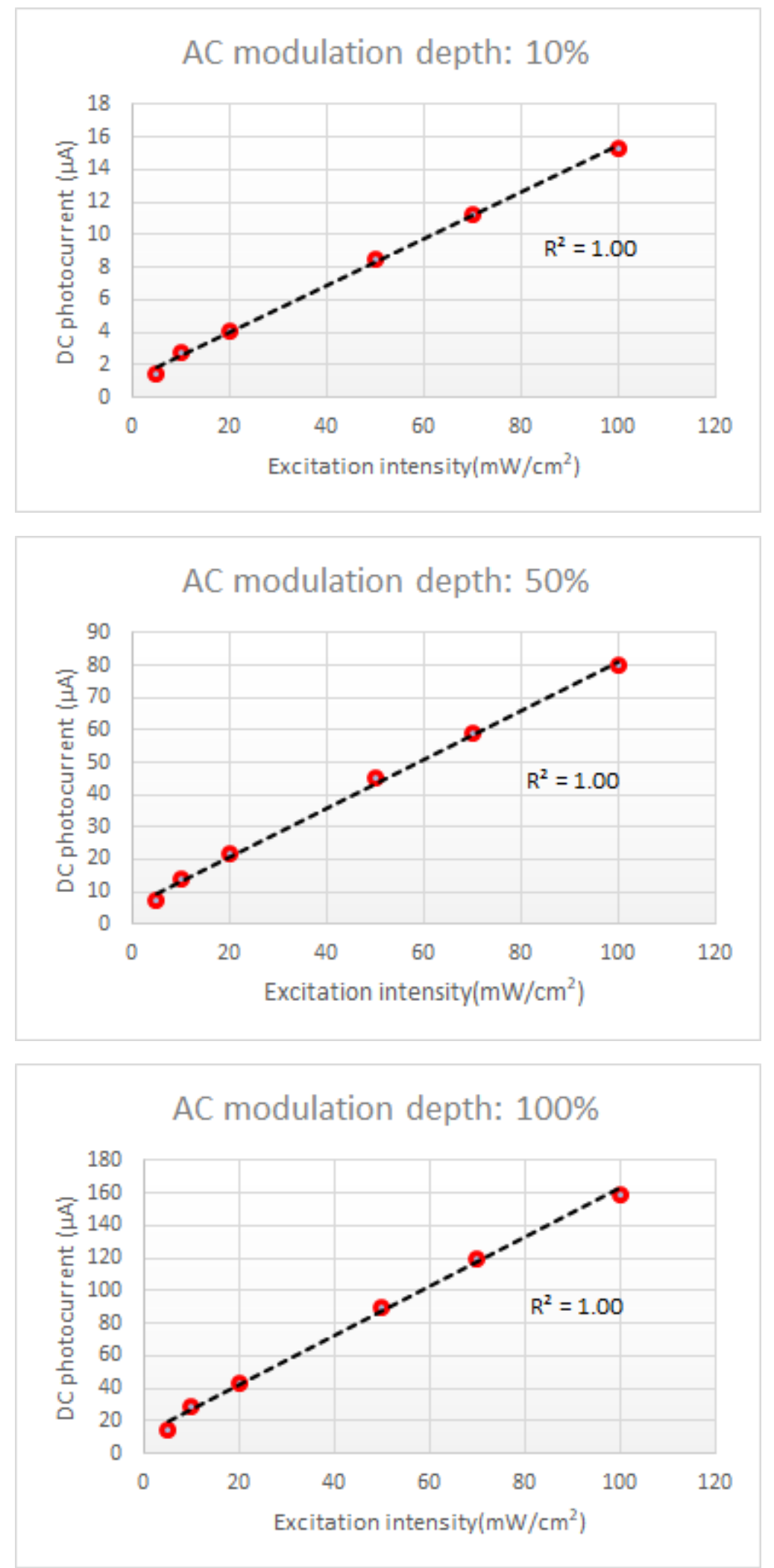

Figure S5: Intensity $\left(5,10,20,50,70\right.$ and $\left.100 \mathrm{mw} / \mathrm{cm}^{2}\right)$ dependent DC photocurrent of a lightly aged PTB7:Coi8DFIC(1:1) device at different depths of AC modulation. 

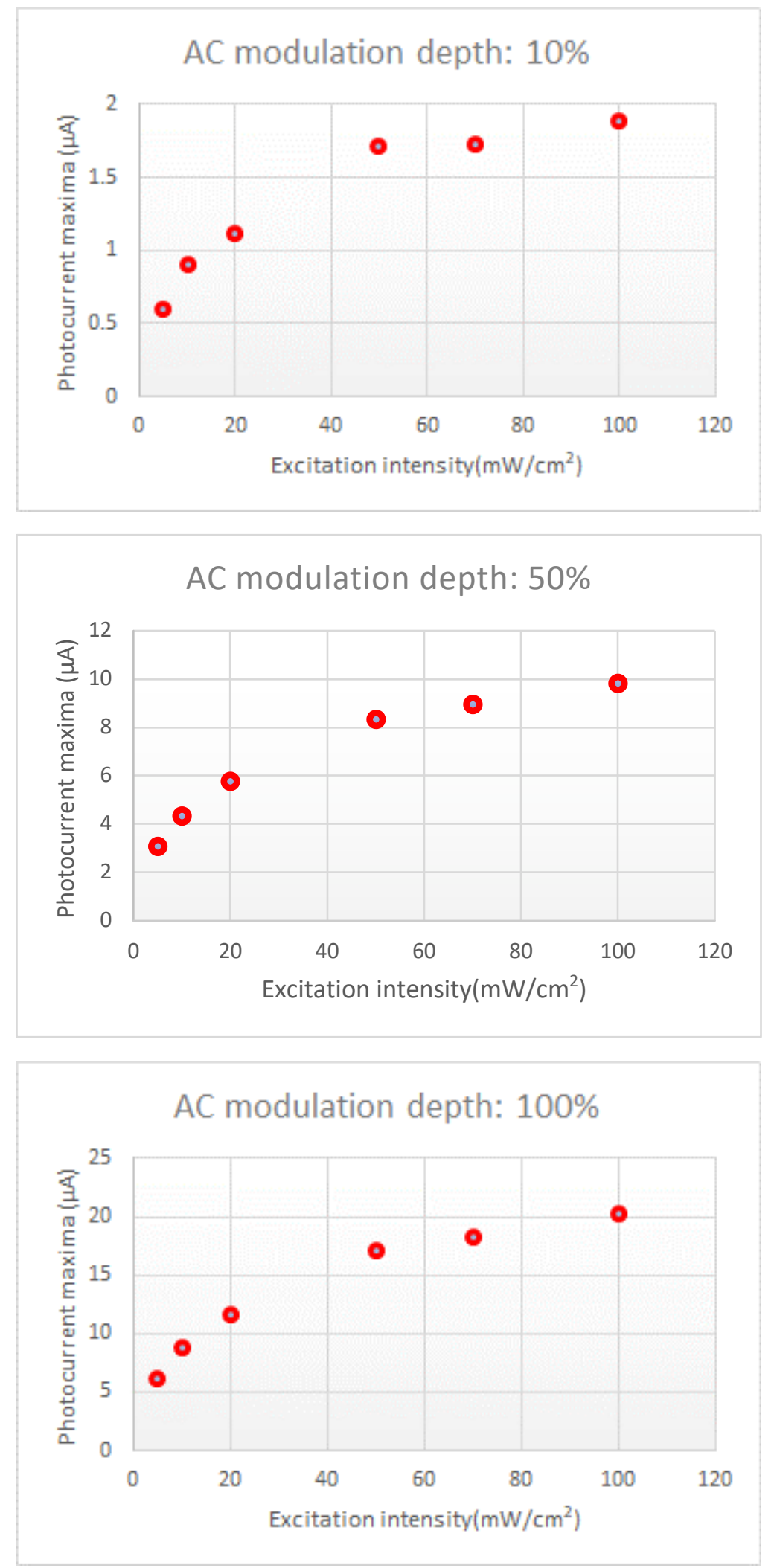

Figure S6: Intensity $\left(5,10,20,50,70\right.$ and $\left.100 \mathrm{mw} / \mathrm{cm}^{2}\right)$ dependent photocurrent gain peak maxima of a heavily aged PTB7:Coi8DFIC(1:1) device at different depths of AC modulation. 

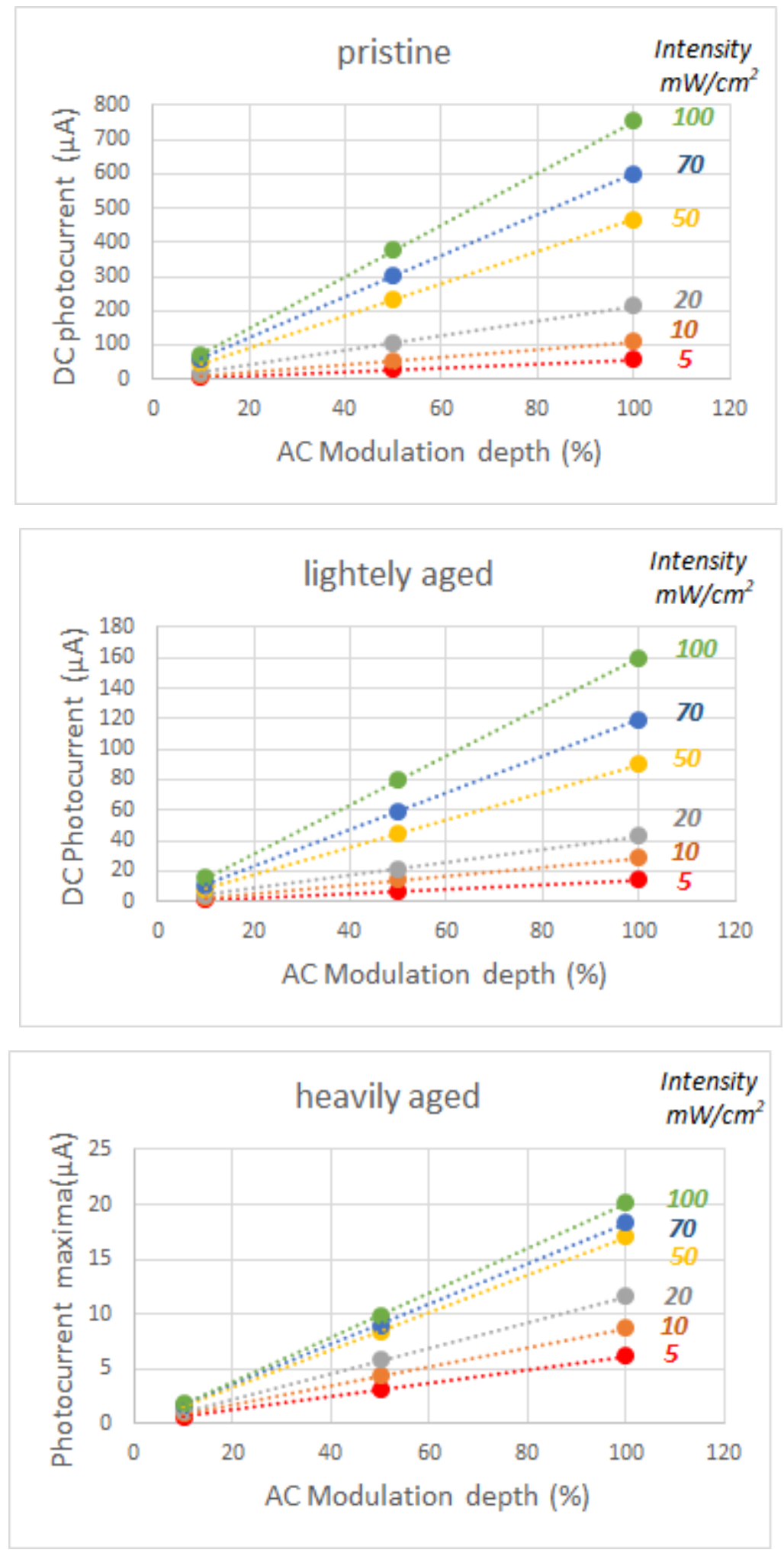

Figure S7: AC modulation depth dependent photocurrent of a pristine, and lightly and heavily aged PTB7:Coi8DFIC(1:1) device at 5, 10, 20, 50, 70 and $100 \mathrm{~mW} / \mathrm{cm}^{2}$. 


\section{$\underline{\text { Raman Spectroscopy }}$}

The details of the Raman instrumentation were described previously ${ }^{1}$. In brief, the donor-acceptor blend was spin coated on a thin glass film and excited with $561 \mathrm{~nm}$ light coming out of a diode pumped solid state laser (Cobolt, $561 \mathrm{~nm}$ ). The back-scattered light was filtered using an edge filter which removes Rayleigh light and the Raman signal was analyzed with a CCD camera.

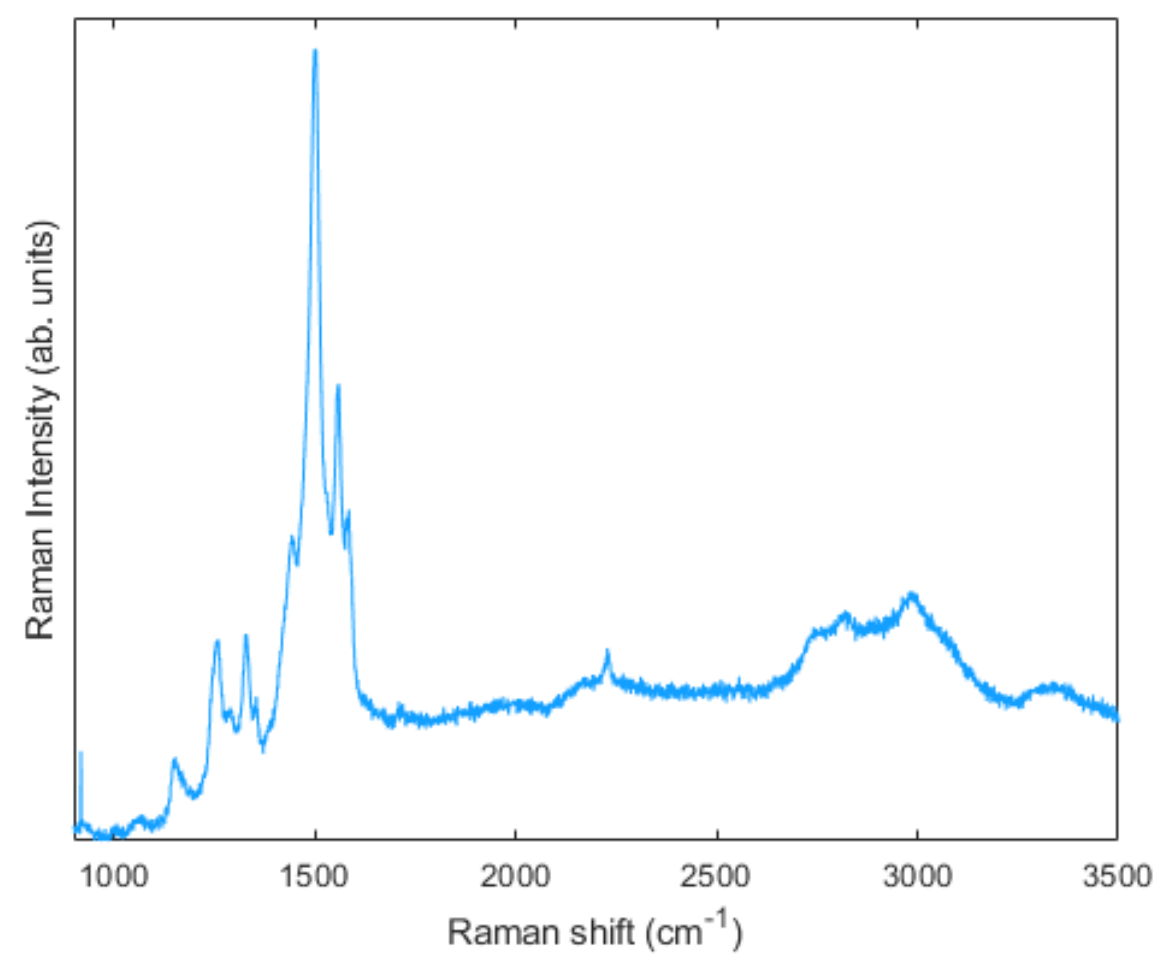

Figure S8: Raman spectrum of PTB7:Coi8DFIC(1:1) thin film.

$\underline{\mathrm{J}-\mathrm{V} \text { parameters of the pristine device }}$

Table S1: Average performance figures of merit obtained from the steady state $\mathrm{J}-\mathrm{V}$ measurement of pristine PTB7:Coi8DFIC(1:1) devices.

\begin{tabular}{|l|l|l|l|}
\hline $\begin{array}{l}\mathrm{V}_{\mathrm{oc}} \\
{[\mathrm{V}]}\end{array}$ & $\begin{array}{l}\mathrm{J}_{\mathrm{sc}} \\
{\left[\mathrm{mA} / \mathrm{cm}^{2}\right]}\end{array}$ & $\begin{array}{l}\mathrm{FF} \\
{[\%]}\end{array}$ & $\begin{array}{l}\mathrm{PCE} \\
{[\%]}\end{array}$ \\
\hline 0.48 & 9.71 & 45.83 & 2.11 \\
\hline
\end{tabular}


Examples of IMPS fits with eq. 1
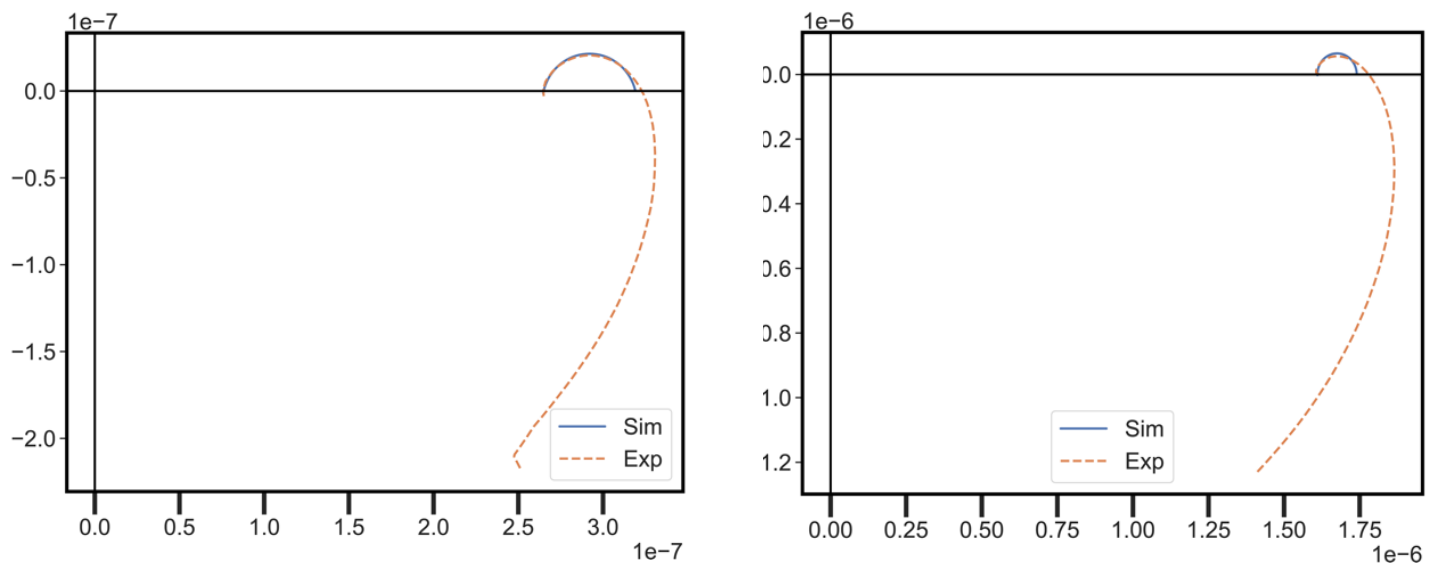

Figure S9. Examples of $1^{\text {st }}$ quadrant IMPS fits for lightly aged devices. Left panel: $1 \mathrm{~mW} / \mathrm{cm}^{2}$; Right panel: $5 \mathrm{~mW} / \mathrm{cm}^{2}$
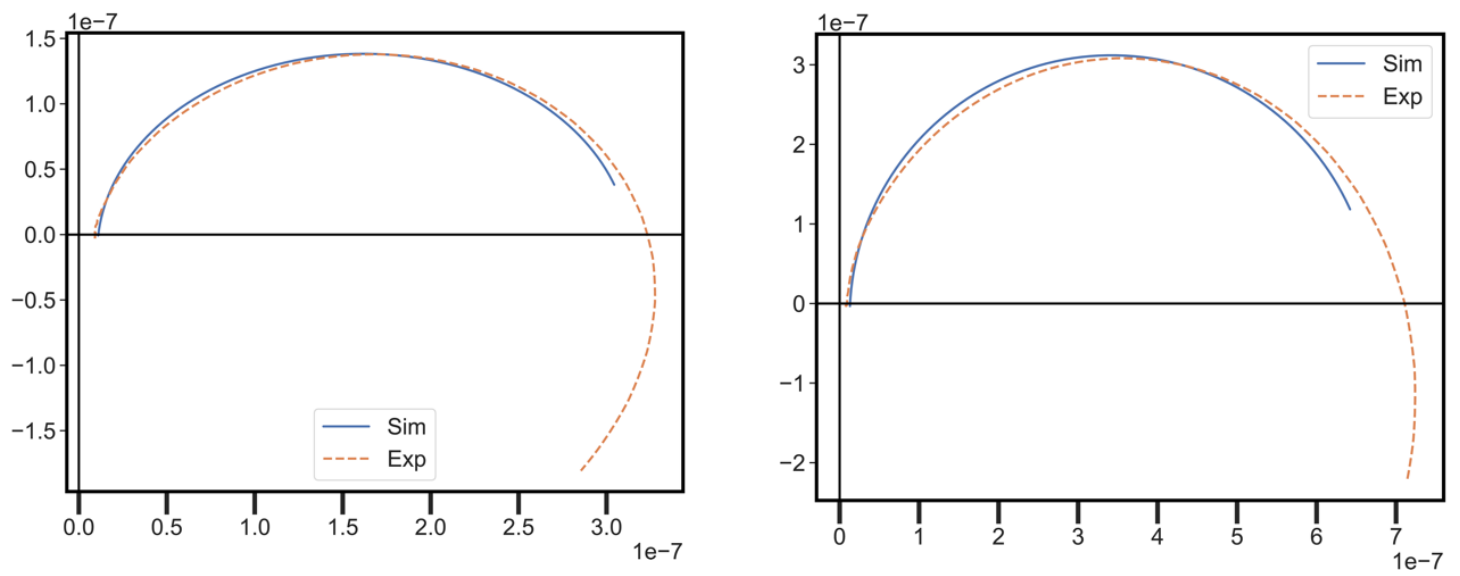

Figure S10. Examples of $1^{\text {st }}$ quadrant IMPS fits for heavily aged devices. Left panel: $7 \mathrm{~mW} / \mathrm{cm}^{2}$; Right panel: $15 \mathrm{~mW} / \mathrm{cm}^{2}$ 

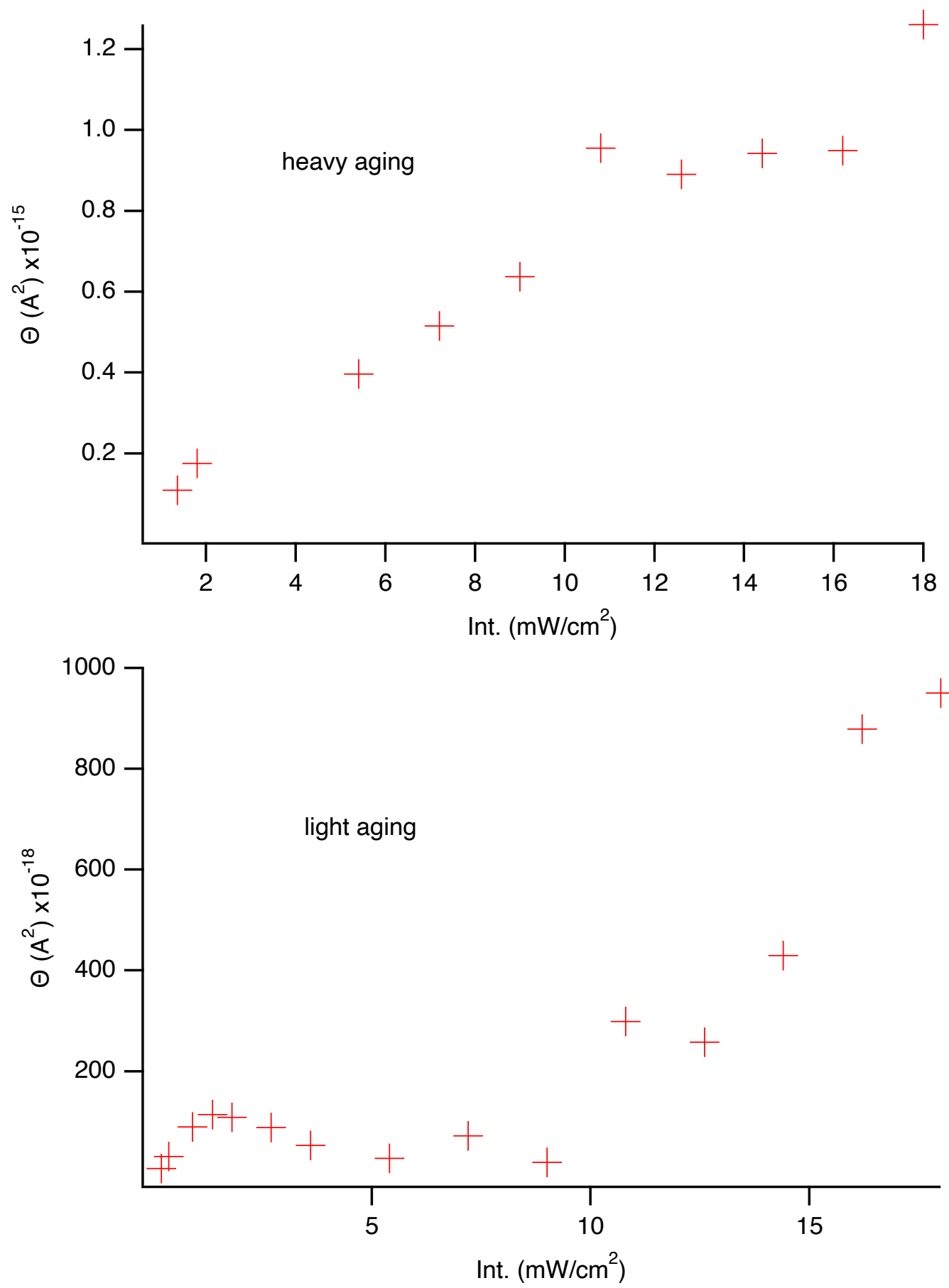

Figure S11. Estimates of error from fitting eq. 1 to experimental $1^{\text {st }}$ quadrant components for light and heavily aged devices.

Fig. S11 displays the error associated with fitting eq. 1 to experimental IMPS data in the $1^{\text {st }}$ quadrant region. This parameter was estimated by, 


$$
\Theta=\frac{1}{N} \sum_{i}\left(\operatorname{Re}\left(J_{\text {model }}^{(i)}\right)-\operatorname{Re}\left(J_{\text {sim }}^{(i)}\right)\right)^{2}+\left(\operatorname{Im}\left(J_{\text {model }}^{(i)}\right)-\operatorname{Im}\left(J_{\text {sim }}^{(i)}\right)\right)^{2}
$$

which considers the mean squared Euclidean distance between the data and the fit where $\mathrm{N}$ is the number of data points fitted in the first quadrant. The fit error parameter has units of $\mathrm{A}^{2}$.

\section{References}

1. Gao, J.; Grey, J. K. Resonance Raman Overtones Reveal Vibrational Displacements and Dynamics of Crystalline and Amorphous Poly(3-Hexylthiophene) Chains In Fullerene Blends. J. Chem. Phys. 2013, 139, 044903. 